\title{
MICROSTRUCTURE AND WEAR-RESISTANT PROPERTIES OF WC/SS316L COMPOSITE COATINGS PREPARED BY SUPERSONIC LASER DEPOSITION
}

\author{
BO LI ${ }^{1,2}$, ZHIHONG LI ${ }^{1,2}$, LIJING YANG ${ }^{1,2}$ and JIANHUA YAO ${ }^{1,2}$ \\ ${ }^{1}$ Research Center of Laser Processing Technology and Engineering, Zhejiang University of Technology \\ 18 Chaowang Str., 310014, Hangzhou, PRC.E-mail: libo1011@zjut.edu.cn \\ ${ }^{2}$ Zhejiang Provincial Collaborative Innovation Center of High-end Laser Manufacturing Equipment \\ 18 Chaowang Str., 310014, Hangzhou, PRC. E-mail: laser@zjut.edu.cn
}

\begin{abstract}
Supersonic laser deposition (SLD) is a newly developed coating method which combines the supersonic powder jet found in cold spray (CS) with synchronous laser heating of the deposition zone. The addition of laser heat energy into CS enables a change in the thermodynamic state of impacting particles and substrate, thereby significantly lowering the critical deposition velocities required for effective coating formation and allowing the range of materials deposited to expand to higher strength materials which are of considerable engineering interest. This paper presents the ability of SLD technique to deposit hard metal matrix composite (MMC) coatings, such as WC/SS316L. The focus of this research is on the comparison between composite coatings produced with conventional CS and those produced with SLD. The microstructure evolution, mechanical deformation mechanisms, correlation between functional properties and process parameters were elaborated in detail. The experimental results show that with the assistance of laser irradiation, WC/SS316L composite coatings can be successfully deposited using SLD. The obtained coatings are superior to that processed with CS, because SLD can improve the deposition efficiency, coating density, interface bonding as compared to CS due to the softening of particle and substrate by laser irradiation. It can be found that SLD is capable of depositing high strength MMC coatings with good quality, thus exhibiting great potential in the field of metal 3D printing. 13 Ref., 1 Table, 8 Figures.
\end{abstract}

Ke y word s : supersonic laser deposition, metal matrix composite coating, microstructure, composition, wear-resistant property

Cold spray (CS) is a rapidly emerging coating technology, in which small powder particles in a solid state are deposited on a substrate via high-velocity impact at temperatures lower than the melting point of the powder material [1-3]. In the CS process, small powder particles $(5-50 \mu \mathrm{m})$ are accelerated to a high velocity in a supersonic gas jet and then imping onto the substrate or already deposited coating to build up a coating. The main advantage of CS is the lower heat input as compared to traditional high-temperature coating methods such as thermal spray and laser cladding. Only thermal input in CS is optional gas heating, which can be used to increase the local speed of sound of the process gas, and its positive drag force on particles. As a consequence, the deleterious effects of high-temperature oxidation, evaporation, melting, crystallization, residual stress, debonding, gas release, and other common problems for traditional thermal spray and laser cladding methods are minimized or eliminated.
Eliminating the adverse effects of high-temperature on coatings and substrates offers significant advantages and new possibilities, and makes CS promising for many industrial applications. However, to achieve the high particle velocities that CS requires for successful deposition, helium is often used as the process gas, which is an expensive, limited resource. Moreover, a gas heater is often employed to heat the process gas as high as $900{ }^{\circ} \mathrm{C}$ to increase gas and particle velocity. The need for hot helium gas results in high operating costs arising from the power consumption for heating the gas and the high price of the gas itself. Additionally, it is challenging to deposit hard material coatings with high bonding strength and coating density solely using CS.

It has become clear that there would be distinct advantages in developing a process that could retain the non-melting deposition mechanism of CS, but reduce deposition costs and improve coating functionality. Such a process, known as supersonic laser deposition

"Based on materials presented at Seventh International Conference «Laser technologies in welding and materials processing», September 14-18, 2015, Odessa, Ukraine. 


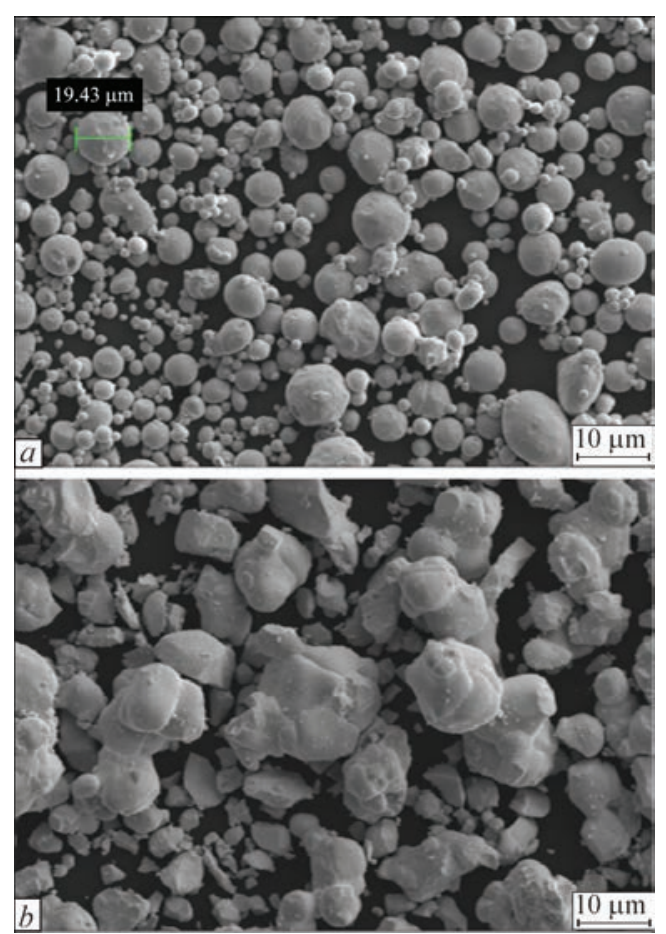

Figure 1. Microscopic images of feedstock materials: $a-$ SS316L; $b-\mathrm{WC}$

(SLD), has been proposed by O'Neill et al. which combine CS with laser heating [4-6]. In SLD, a laser is employed to heat the deposition zone of CS in order to soften both substrate and powder particles, allowing the particles to deform and build up a coating at impact velocities around half of that in CS. Eliminating the need for high impacting velocities permits cold or slightly heated nitrogen to be used instead of high-temperature helium as the process gas, thus reducing operating costs by over an order of magnitude. Furthermore, the SLD technique greatly expands the material range of particle and substrate which can be processed due to the reduction of critical deposition velocity. SLD has been applied to fabricate a variety of material coatings such as copper, titanium, Stellite 6, Ni60, Al-Cu, Al-Si alloys and so on [7-12].

Metal matrix composites (MMCs), which are generally composed of a hard reinforcing non-metallic material and a metallic matrix, are a useful class of materials for surface coating that enable physical properties such as density and thermal/electrical conductivity to be tailored, along with mechanical properties such as strength and wear resistance. Currently, laser cladding, thermal spray and CS are the most widely used methods for preparing MMC coatings. The high-temperature involved in laser cladding and thermal spray would lead to porosity, microcrack formation, dilution, phase transformation, high thermal stress, etc. The main problem for cold-sprayed MMC coatings is the bonding strength, because the metallic matrix is often soft materials such as $\mathrm{Al}, \mathrm{Cu}, \mathrm{Ni}$, which results in weak holding force between the metallic matrixes and reinforcing particles. As a result, the reinforcing particles are prone to spall off from the coating in the subsequent real applications, which would degrade the coating's performance.

Based on the characteristics of SLD, it is expected to deposit hard material coatings by this process at a lower impact velocity with high coating density and strong interfacial boding. Meanwhile, the deleterious effects of high-temperature coating methods could be effectively avoided due to the relatively low-temperature deposition feature of SLD. Currently, most of the research reported in literature about SLD technique focused on single material coatings and seldom involved MMC coatings. Therefore, in this study, the SLD process was adopted to deposit tungsten carbide reinforced stainless steel (WC/SS316L) composite coatings onto carbon steel substrate in order to demonstrate the feasibility of SLD technique to prepare hard metallic matrix composite coatings. For comparison, the cold-sprayed composite coatings were also produced under the same conditions except that laser heating was not involved. The microstructure, composition, interface bonding, coating density and wear-resistant properties of the as-deposited composite coatings were investigated using scanning electron microscopy (SEM), optical microscopy (OM), $\mathrm{X}$-ray diffraction (XRD) and pin-on-disc wear tester.

Experimental section. Feedstock materials. Commercially available stainless steel (SS316L) powder and tungsten carbide (WC) powder were used in the as-received conditions as feedstock materials. The particles' shapes of SS316L and WC powder are spherical and irregular (Figure 1). The composite powder consisting of $30 \mathrm{vol} . \% \mathrm{WC}$ and $70 \mathrm{vol} . \%$ SS316L was mechanically milled in a cylinder rotating at speed of $200 \mathrm{rpm}$ for $2 \mathrm{~h}$. The ball milling process was used to obtain evenly mixed composite powder, which would be beneficial for depositing composite coatings with uniformly distributed reinforcing particles. The substrate material was carbon steel and the substrate specimens had dimension of $100 \times 60 \times 10 \mathrm{~mm}$. Before the coating process, the substrate surface was grit-blasted using 24 mesh alumina and ultrasonic cleaned in alcohol.

SLD system and coating process. The schematic diagram of the SLD system used in this study is illustrated in Figure 2, $a$. High-pressure gas was supplied to a converging-diverging nozzle in two different imports: one was through the gas heater; the other was via a powder feeder where feedstock powders were held. The feedstock powder stream and high-pressure gas were mixed and passed through the nozzle 
where the particles were accelerated to supersonic speed. The high-velocity particles impacted a region of the substrate which was synchronously heated by diode laser (Laserline LDF 400-1000, Germany) with 960-980 nm wavelengths and $4 \mathrm{~kW}$ maximum power. Combined lenses were used to focus the laser beam onto the substrate surface, and the diameter of the laser spot was $5 \mathrm{~mm}$. A high-speed infrared pyrometer was used to obtain real-time temperature measurements and control the temperature of the deposition zone (referring to as «deposition temperature» hereafter) during the SLD process. Data from the pyrometer was fed through a closed-loop feedback system which altered laser power as necessary to maintain the desired temperature. The nozzle, laser head and pyrometer were assembled on robot (STAÜBLI TX 90 , Switzerland). The spraying nozzle was perpendicular to the substrate surface. The laser beam was at angle of $30^{\circ}$ to the surface normal. The laser energy and powder distribution are schematically illustrated in Figure 2, $b$. In the deposition process, the substrate was stationary and the nozzle, laser head and pyrometer were moveable, controlled by the robot. The process gas was nitrogen. The process parameters for WC/SS316L composite coatings except deposition temperature were kept constant at the optimal values: gas pressure $2.5 \mathrm{MPa}$; gas temperature $400{ }^{\circ} \mathrm{C}$; laser traverse speed $30 \mathrm{~mm} / \mathrm{s}$; powder feeding rate $40 \mathrm{~g} / \mathrm{min}$; spray distance $30 \mathrm{~mm}$.

Coating characterization. The coating specimens were cross-sectioned perpendicular to the coating surface with electric discharge wire cutting, followed by conventional metallography preparation procedures. An aqueous solution, consisting of $45 \mathrm{ml}$ of $\mathrm{HCl}, 15 \mathrm{ml}$ of $\mathrm{HNO}_{3}$ and $20 \mathrm{ml}$ of methanol, was used for etching. A field emission scanning electron microscopy (SIGMA HV-01-043, Carl Zeiss) and optical microscopy (Axio Scope, A1, Zeiss) were used to analyse the coating's microstructure and interface bonding. Backscatter SEM images were taken to maximize contrast between the WC particles and stainless steel matrix. The phase of the composite coatings were investigated using X-ray diffractometer (D8 Advance, Brucker) with $\mathrm{CuK} K_{\alpha}$ radiation, at $45 \mathrm{kV}, 40 \mathrm{~mA}$, scanning rate of $0.02 \mathrm{deg} / \mathrm{s}$. Pin-on-disc wear test was conducted on the composite coatings at room temperature under a dry-lubricating condition. The pin was $4 \mathrm{~mm}$ diameter $\mathrm{Si}_{3} \mathrm{~N}_{4}$ ceramic ball, having the hardness of $H V 2200$ (HRC 90). The disc was the tested specimen which was polished, cleaned in a ultrasonic bath, and finally dried. The test was performed under normal load of $500 \mathrm{~g}$ at rotational speed of $500 \mathrm{rpm}$ of the tested specimen between the contacting surfaces; sliding duration was $60 \mathrm{~min}$.

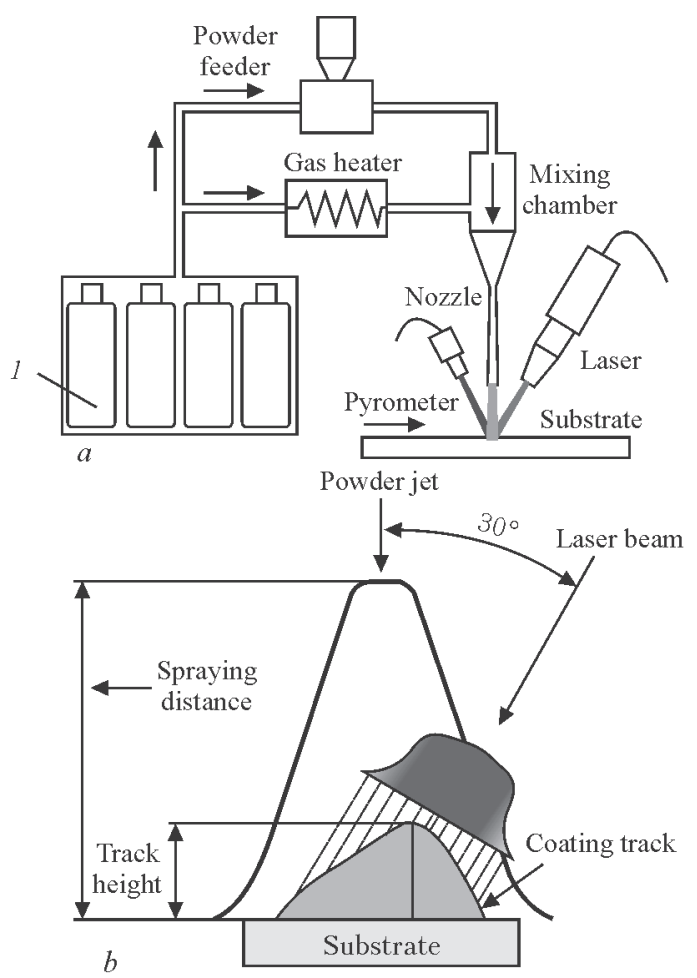

Figure 2. Schematic illustration of SLD system (a), laser energy and powder distribution in the SLD process $(b)$

Results and discussion. Deposition efficiency. The deposition efficiencies (DE) of the WC/SS316L composite coatings deposited at different deposition temperature were compared with respect to coating layer thickness. Shown in Figure 3 are the $\mathrm{OM}$ images of cross section of the coating specimens. As can be seen, the central peak heights of the as-deposited coatings are gradually increased with elevating the deposition temperature. The peak height of the WC/SS316L coating deposited without laser assistance is $734.04 \mu \mathrm{m}$ while it is increased by $43 \%$ to $1050.91 \mu \mathrm{m}$ as $T_{\mathrm{d}}=800{ }^{\circ} \mathrm{C}$ was employed, indicating that $\mathrm{DE}$ can be improved with increasing the deposition temperature.

The improvement of DE should be ascribed to the reduction of critical deposition velocity due to the softening of spraying particles by laser irradiation. One of the most important parameter in the CS process is the critical deposition velocity. For a given material, there exists a critical deposition velocity that must be achieved. Only particles, velocities of which exceed this value, can be effectively deposited, in turn producing the desired coating. Conversely, particles that have not reached this threshold velocity contribute to the erosion of the substrate. Theoretical modelling of critical deposition velocity $(\mathrm{m} / \mathrm{s})$ proposed by Assadi et al. can be expressed as [13]:

$$
v_{\mathrm{cr}}=667-14 \rho+0.08 T_{\mathrm{m}}+0.1 \sigma_{\mathrm{t}}-0.4 T_{\mathrm{i}} \text {, }
$$



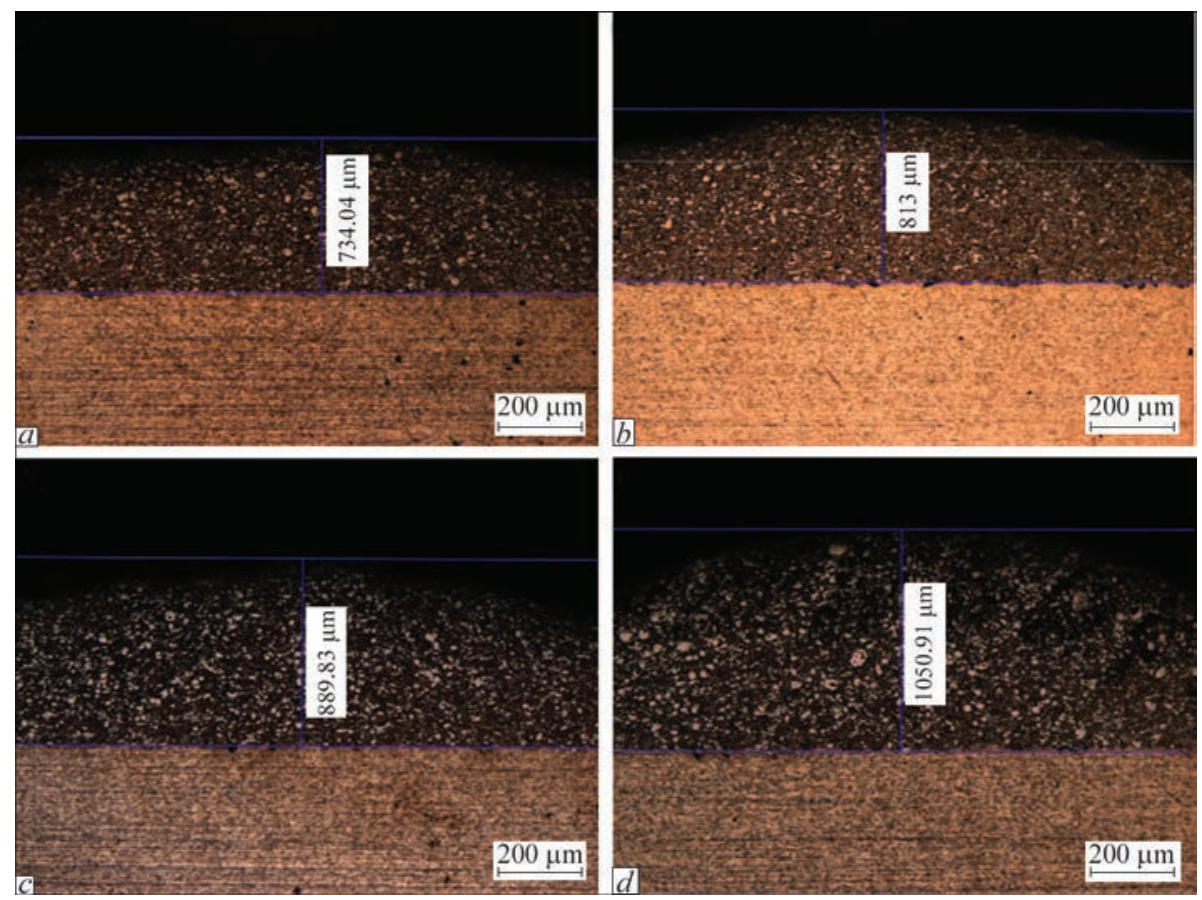

Figure 3. Coating thickness prepared at different deposition temperature: $a-$ ambient temperature; $b-T_{\mathrm{d}}=400 ; c-600 ; d-$ $800^{\circ} \mathrm{C}$

where $\rho$ is the density of the material, $\mathrm{g} / \mathrm{cm}^{3} ; T_{\mathrm{m}}$ is the melting temperature, ${ }^{\circ} \mathrm{C} ; \sigma_{\mathrm{t}}$ is the ultimate strength, $\mathrm{MPa} ; \mathrm{T}_{\mathrm{i}}$ is the initial particle temperature, ${ }^{\circ} \mathrm{C}$. According to formula (1), particle preheating will decrease the critical deposition velocity because as $T_{\mathrm{i}}$ is increased, $\sigma_{\mathrm{t}}$ is reduced. Both the increase of $T_{\mathrm{i}}$ and the reduction of $\sigma_{t}$ would contribute to the decrease of $v_{\text {cr. }}$

In this study, the spraying nozzle was perpendicular to the substrate surface and laser beam was at angle of $30^{\circ}$ to the surface normal (see Figure 2, b). The powder jet and laser beam partially overlapped with each other. Although the spraying particles were travelling at high velocities and had limited time of exposure to the laser, it is expected that the particles would be significantly heated in flight by laser prior to hitting the substrate because of the high laser power density and small particle size, which could bring down the critical deposition velocity of spraying particles. As a consequence, the proportion of particles exceeding this velocity would increase, leading to the improvement in DE (see Figure 3).

Distribution and content of reinforcing particles in the composite coatings. It can be seen that WC particles are evenly distributed in all the coating specimens. Therefore, we randomly selected one area in each coating and calculated the WC area fraction in these selected areas using image analysis software to evaluate the dependence of WC content on deposition temperature. The calculated results are illustrated in Figure 4. It is shown that the area fraction of WC particles in the CS coating is about $22.55 \%$ and it increases slightly to $24.42 \%$ when $T_{\mathrm{d}}=400{ }^{\circ} \mathrm{C}$ is adopt- ed. As the deposition temperature is further increased to $600{ }^{\circ} \mathrm{C}$, the area fraction of WC particles increases greatly to $28.55 \%$. The coating deposited at $800{ }^{\circ} \mathrm{C}$ has about $29.29 \%$ area fraction of WC particles, which is very close to the volume fraction $(30 \%)$ of WC particles in the original composite powder. This indicates that laser heating benefits the effective deposition of WC particles in the composite coatings.

Cold-sprayed MMC coatings generally consist of pure metallic matrix and ceramic reinforcing particles, such as $\mathrm{Al} / \mathrm{AlN}, \mathrm{Ni} / \mathrm{Al}_{2} \mathrm{O}_{3}, \mathrm{Cu} / \mathrm{Al}_{2} \mathrm{O}_{3}$ etc. During the $\mathrm{CS}$ process, the reinforcing particles cannot deform due to high hardness and they need to be embedded in the deformable metallic matrix to form composite coating. Therefore, the plastic deformation ability of metallic matrix plays a decisive role in the formation of MMC coatings. In this study, without laser assistance or with less laser heating, the SS316L powder wasn't softened enough to accommodate the hard particles, resulting in relatively low WC content. In the case of more laser heating, the SS316L powder easily deformed to take in WC particles owing to sufficient softening, resulting in higher WC particle concentration (see Figure 4).

Coating density. According to the previous results, it can be found that the composite coating deposited at $800^{\circ} \mathrm{C}$ has relatively high DE and WC content. Therefore, the following comparative studies mainly focus on this sample and CS sample. Shown in Figure 5 is the comparison of coating density between these two samples. It can be seen from Figure 5, a that there are lots of gaps and pores in the CS coating, 

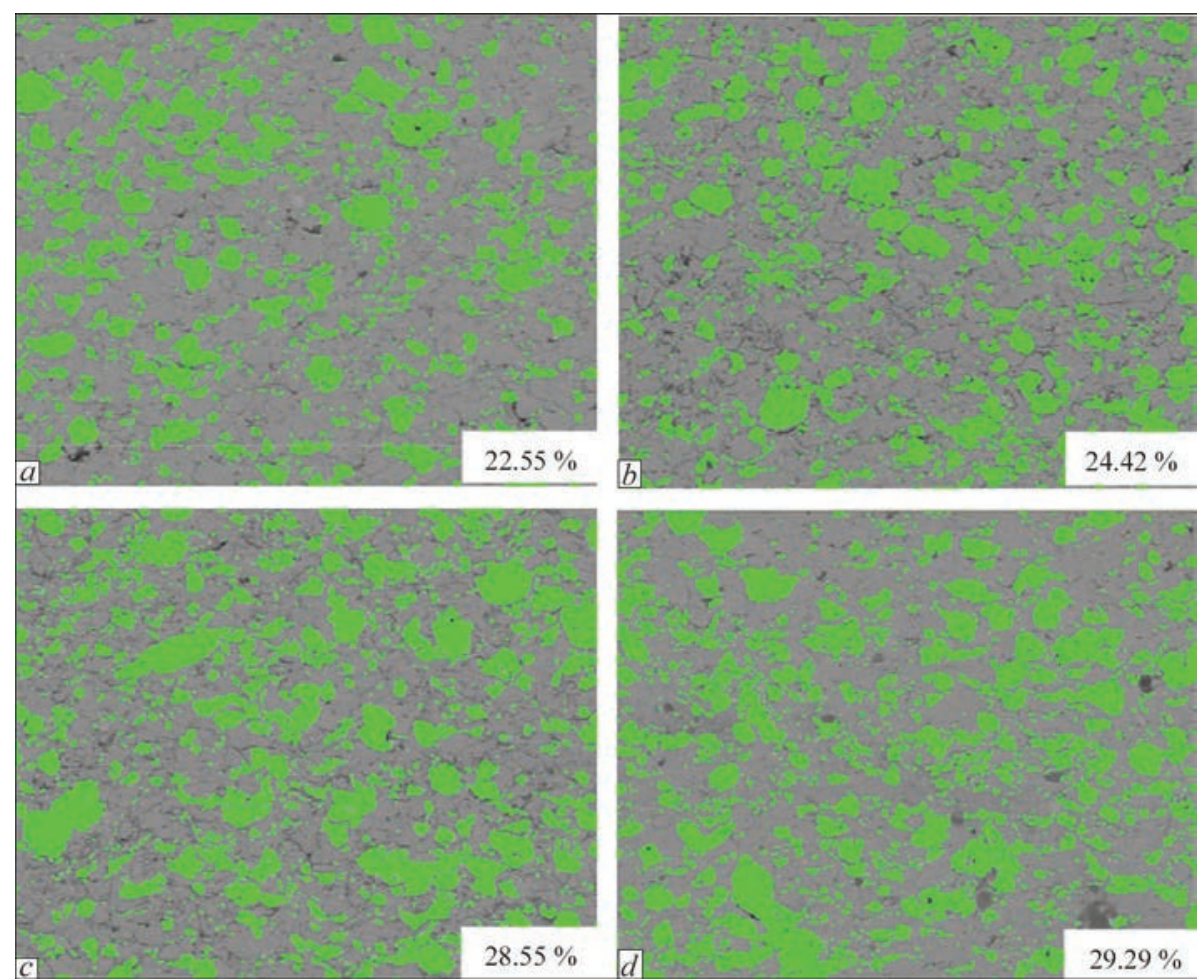

Figure 4. WC distribution and content in as-deposited coatings at different deposition temperatures: $a$ - ambient temperature; $b-$ $T_{\mathrm{d}}=400 ; c-600 ; d-800^{\circ} \mathrm{C}$

which indicates poor bonding strength between powder particles and low density of this coating. Moreover, evident holes can also be clearly observed in the CS coating (see Figure 5,a). The appearance of these holes arises from the debonding of the fractured WC particles. With the assistance of laser heating, improved coating density can be obtained for the as-deposited coating. Gaps and pores are hardly observed in the SLD coating, along with the disappearance of the holes. These results imply that laser irradiation has beneficial effects on the coating density. Bray et al. [4] compared the porosity of titanium coating prepared by CS, high-velocity oxygen fuel spray (HVOF) and SLD respectively. Their results revealed that the porosity level of the CS and HVOF coatings were 3.1 and $5.4 \%$ respectively, while that of the SLD coating was only about $0.5 \%$, suggesting that the coating density of SLD coating is superior to that of CS and HVOF ones.

The poor density of CS coating are mainly attributed to the limited plastic deformation of the spraying particles. In the SLD process, the synchronous laser irradiation can effectively soften the spraying particles which would in turn result in sufficient deformation of spraying particles. The sufficiently deformed particles would interlock with each other to form intimate contact, thus showing high coating density. Furthermore, due to the softening of the metallic matrix, hard WC particles wouldn't fracture and spall off from the composite coating as embedding into the metallic matrix, which ensures no holes formed in the SLD coating.

Phase analysis. The influence of laser heating on phases of the WC/SS316L composite coatings was analysed using XRD. The XRD results for the composite CS and SLD coatings are presented in Figure 6 . It is found that the coating specimens with and

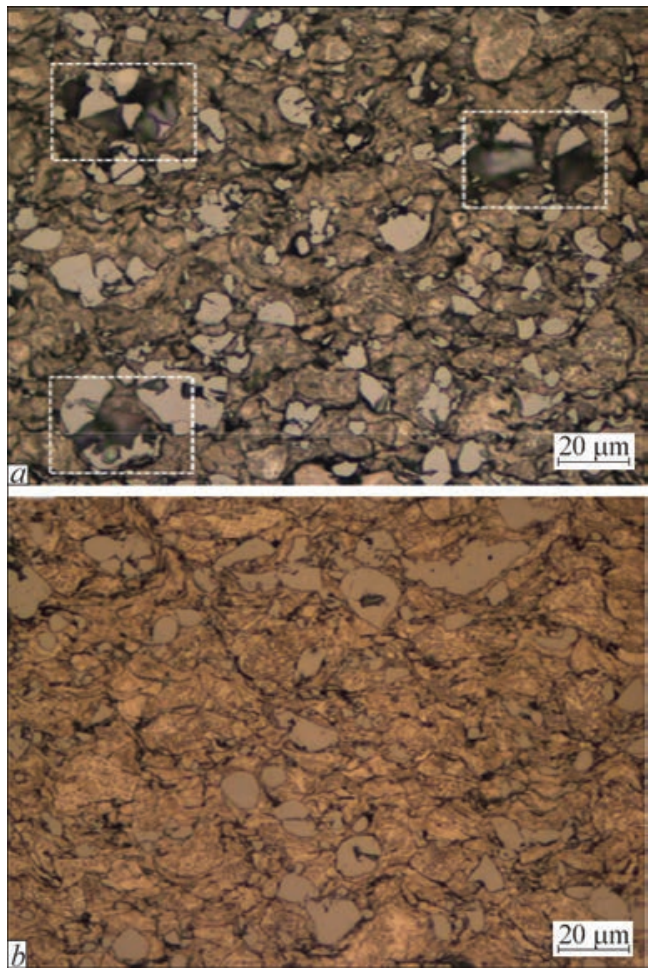

Figure 5. Density of coating prepared by CS (a) and SLD (b) 


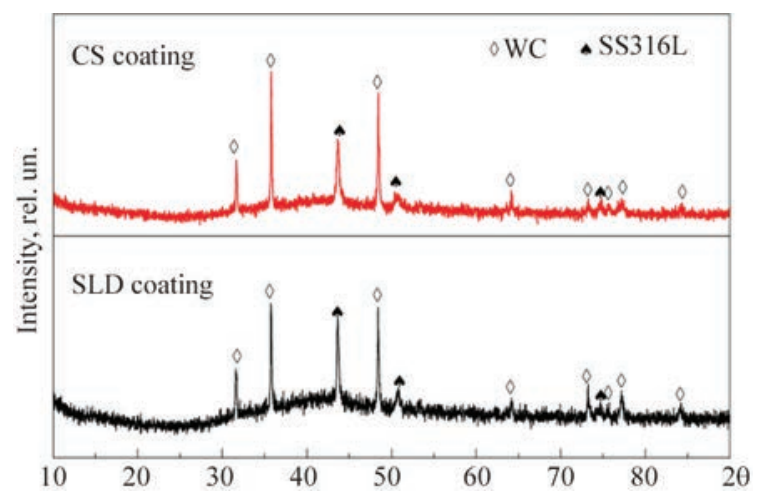

Figure 6. XRD patterns of the CS and SLD composite coatings without laser irradiation have identical phases that are composed of SS316L and WC, implying that SLD still remains solid state deposition mechanism as CS does although laser heating is introduced in this process. In SLD process, the laser power is elaborately controlled to only soften both the spraying particles and the substrate, not melting them. The particles remained solid during flight and deposition and were subject to laser irradiation for only a limited period of time. Thus, the chemical composition and phases did not transform in the SLD coatings as compared to the CS coating, even for the low melting temperature materials such as copper and aluminum. It was found in our previous study [9] that the oxidation of SLD-Cu coating was trivial and can be neglected with comparison to the CS-Cu coating. The relatively low temperature deposition feature of SLD can effectively avoid high thermal stress, oxidation, phase transformation and grain growth in the as-deposited coatings, making it a superior coating technology over thermal spray and laser cladding.

Wear-resistant performance. The evolution of friction coefficient of the test samples were recorded during the wear processes, and the variations of friction coefficient with sliding time are plotted in Figure 7. It is shown that the friction coefficient of the SLD sample is smaller and more stable than that of the CS sample. The average friction coefficient of the

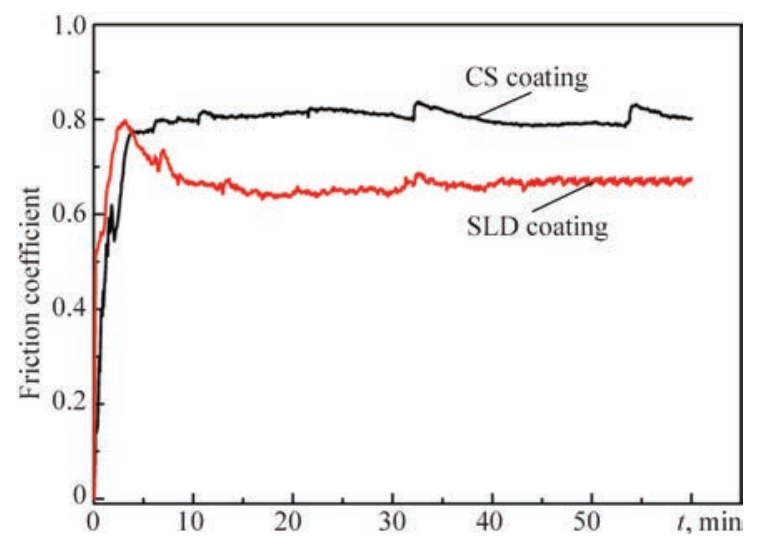

Figure 7. Variations of friction coefficient with sliding time of WC/SS316L composite coatings prepared by CS and SLD
EDS results of worn surfaces of the WC/SS316L composite coatings prepared by CS and SLD

\begin{tabular}{ccccccc}
\hline $\begin{array}{c}\text { Area } \\
\text { number }\end{array}$ & $\mathrm{O}$ & $\mathrm{Si}$ & $\mathrm{Cr}$ & $\mathrm{Fe}$ & $\mathrm{Ni}$ & $\mathrm{W}$ \\
\hline 1 & 11.68 & 1.49 & 12.95 & 57.05 & 7.76 & 9.07 \\
2 & 39.38 & 8.76 & 5.87 & 24.55 & 3.53 & 17.91 \\
3 & 7.32 & 3.63 & 3.71 & 12.8 & 2.50 & 70.04 \\
4 & 36.36 & 10.65 & 5.44 & 23.42 & 4.27 & 19.86 \\
\hline
\end{tabular}

SLD sample is around 0.65 after 60 min sliding, while that of the CS specimen is about 0.8 at the same time.

To further investigate the wear-resistant performance of the WC/SS316L composite coatings, the worn surfaces of the tested samples were examined using SEM, the results are shown in Figure 8. It can be seen that in both the worn surfaces, there are two distinct areas, dark and light. The dark area is more extensive in the CS coating than in the SLD coating. The Table are the EDS results of the dark and light areas in the wear track of CS and SLD coatings. It is shown that the dark areas ( 2 and 4 ) contain a high content of oxygen; in contrast, a lower oxygen content is detected in the light areas (1 and 3). This suggests that oxidation had occurred in the worn track when the coating samples were under the sliding wear tests, and the dark areas were oxidation scales formed during the wear tests. The more oxidation scales in the worn surface of the CS coating indicate, more friction heat was generated in its surface during wear, which
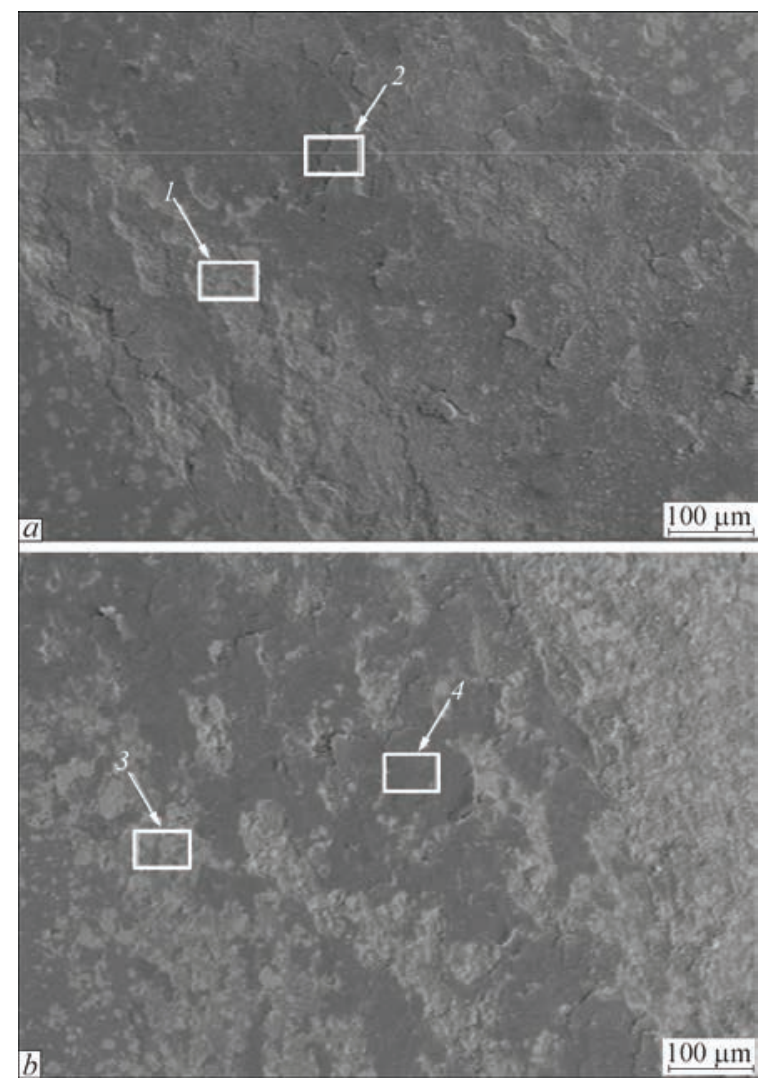

Figure 8. SEM images of worn surfaces of the WC/SS316L composite coatings prepared by CS $(a)$ and SLD $(b)$ 
should be ascribed to the relatively high friction coefficient (see Figure 7).

It can be summarized from the wear test results that the $\mathrm{WC} / \mathrm{SS} 316 \mathrm{~L}$ composite coating produced by SLD has better wear-resistant performance than the CS coating. It is believed that the friction between the contact surfaces was reduced due to the abrasion resistance of hard irregular WC particles. The better wear-resistant properties of the WC/SS316L coating produced by SLD are resulted from the relatively high content of WC particles in the composite coating and the strong interfacial bonding between the WC particles and SS316L matrix due to the beneficial effects of laser irradiation.

\section{Conclusion}

The WC/SS316L composite coatings on carbon steel are successfully prepared in a range of deposition temperatures with the SLD technique, which combines cold spray with laser irradiation. The deposition efficiencies of the SLD coatings are increased with elevating the deposition temperature due to the reduction of critical deposition velocity which results from the softening of spraying particles by laser heating. The WC content and interfacial bonding strength of the coatings are also improved with increasing the deposition temperature, which would in turn significantly improve the wear-resistant performance of the SLD coating. The introduction of laser irradiation into CS doesn't induce phase transformation in the SLD process due to the relatively low temperature deposition feature.

Acknowledgements. The authors would like to appreciate financial supports from the National Natural Science Foundation of China (51475429), the Zhejiang Provincial Commonweal Technology Applied
Research Project (2014C31122) and the Postdoctoral Scientific Research Project of Zhejiang Province (Z42102002).

1. Papyrin, A., Kosarev, V., Klinkov, S. et al. (2007) Cold spray technology, 1-32. Elsevier.

2. Champagne, V.K. (2007) The cold spray materials deposition process. Fundamentals and applications, 11-41. Woodhead Publ.

3. Maev, R.G., Leshchynsky, V. (2008) Introduction to low pressure gas dynamic spray, $1-10$. Wiley-VCH.

4. Bray, M., Cockburn, A., O’Neill, W. (2011) The laser-assisted cold spray process and deposit characterization. Surface and Coating Techn., 203, 2851-2857.

5. Lupoi, R., Sparkes, M., Cockburn, A. et al. (2011) High speed titanium coating by supersonic laser deposition. Materials Letter, 65, 3205-3207.

6. Jones, M., Cockburn, A., Lupoi, R. et al. (2014) Solid-state manufacturing of tungsten deposits onto molybdenum substrates with supersonic laser deposition. Ibid., 134, 295-297.

7. Olakanmi, E.O., Doyoyo, M. (2014) Laser assisted cold-spray corrosion- and wear-resistant coatings: A review. J. Thermal Spray Techn., 23, 765-785.

8. Tlotleng, M., Akinlabi, E., Shukla, M. et al. (2015) Microstructural and mechanical evaluation of laser-assisted cold sprayed bio-ceramic coatings: Potential use for biomedical applications. Ibid., 24, 423-435.

9. Li, B., Yang, L.J., Li, Z.H. et al. (2015) Beneficial effects of synchronous laser irradiation on the characteristics of coldsprayed copper coatings. Ibid., 24, 836-847.

10. Yao, J.H., Yang, L.J., Li, B. et al. (2015) Beneficial effects of laser irradiation on the deposition process of diamond/Ni60 composite coating with cold spray. Appl. Surface Sci., 330, 300-308.

11. Luo, F., Cockburn, A., Cai, D.B. et al. (2015) Simulation analysis of Stellite 6 particle impact on steel substrate in supersonic laser deposition process. J. Thermal Spray Techn., 24, 378-393.

12. Luo, F., Cockburn, A., Lupoi, R. et al. (2012) Performance comparison of Stellite 6 deposited on steel using supersonic laser deposition and laser cladding. Surface and Coatings Techn., 212, 119-127.

13. Assadi, H., Gartner, F., Stoltenhoff, H. et al. (2004) Bonding mechanism in cold gas spray. Acta Materialia, 51, 4379-4394. 